\title{
Disponibilidad de Variedades de Pastos y Forrajes como Potenciales Materiales Lignocelulósicos para la Producción de Bioetanol en Colombia
}

\author{
Eliana M. Cardona ${ }^{(1)}$, Luis A. Rios ${ }^{(1) *}$ y Juan D. Peña ${ }^{(2)}$ \\ (1) Universidad de Antioquia, Grupo Procesos Fisicoquímicos Aplicados, Departamento de \\ Ingeniería Química, Calle 62 № 52-59, Medellín, Colombia. (e-mail: larios@udea.edu.co) \\ (2) Investigación y Desarrollo Negocios Energía, Empresas Públicas de Medellín, E.S.P. Carrera \\ 58 № 42-125, Medellín, Colombia (e-mail: Juan.Pena.Alvarez@epm.com.co). \\ * autor a quien debe ser dirigida la correspondencia.
}

Recibido Mar. 23, 2012; Aceptado May. 16, 2012; Versión final recibida Jul. 10, 2012

\begin{abstract}
Resumen
Se presenta una revisión sobre las variedades más comunes de pastos y forrajes en Colombia para analizar su potencial como materiales lignocelulósicos para la producción de combustibles como bioetanol y biobutanol. Para ello se considera ubicación, rendimiento de producción por hectárea y por año, composición lignocelulósica y rendimiento a etanol, como características principales para determinar su potencial uso. Como resultado, se presentan los pastos elefante y king como los más apropiados para su exploración en procesos de producción de biocombustibles, con rendimientos de materia verde por hectárea al año entre 360 y 400 toneladas y entre 240 y 360 toneladas respectivamente. Además, con base en su concentración de celulosa y hemicelulosa pueden obtenerse rendimientos teóricos de 466,9 litros de etanol por tonelada seca de pasto elefante y 449,7 litros de etanol por tonelada seca de king grass.
\end{abstract}

Palabras clave: pastos y forrajes, composición lignocelulósica, rendimiento a etanol, bioetanol

\section{Availability of Grasses and Forages as Potential Lignocellulosic Materials for Bioethanol Production in Colombia}

\begin{abstract}
A review on the most common species of grasses and forages in Colombia to determine their potential use as lignocellulosic materials for the production of fuels like bioethanol and biobutanol is presented. For this, location, production yield per hectare and per year, lignocellulosic composition and ethanol yield are considered as main characteristics for determining their potential use. As result, elephant grass and king grass, are presented as the most appropriate ones for their exploration in biofuel production processes with green forages yields per acre per year between 360 and 400 ton and between 240 and 360 ton, respectively. Furthermore based in its cellulose and hemicellulose concentration, theoretical ethanol yields of $466,9 \mathrm{~L} / \mathrm{dry}$ ton of elephant grass and 449,7 L/dry ton of king grass are obtained.
\end{abstract}

Keywords: grasses and forages, lignocellulosic composition, ethanol yield, bioethanol. 


\section{INTRODUCCIÓN}

En Colombia, los recursos naturales existentes son abundantes e incluso desconocidos en cuanto a sus valores, usos y manejo, por lo cual no son aprovechados de manera óptima y eficiente para satisfacer las diferentes necesidades generadas por el constante desarrollo y crecimiento económico del país. En Colombia, la producción de pastos y forrajes está enfocada principalmente en la producción ganadera, y la información reportada en cuanto a existencia, zonas sembradas, toneladas cultivadas, etc., es bastante escaza.

Las especies forrajeras se dividen principalmente en gramíneas y leguminosas. Las gramíneas comprenden aproximadamente $75 \%$ de las plantas forrajeras, existen 700 géneros de gramíneas con 10.000 especies de las cuales son importantes 40; clasificadas por zonas, 25 son de la zona templada, 9 de la zona tropical y 6 de diferente origen (Ministerio De Agricultura Y Desarrollo Rural 1992). Las leguminosas que crecen espontáneamente en Colombia agrupan 23 géneros con 73 especies; este orden botánico tiene una marcada trascendencia ya que en asocio con las gramíneas son los grupos de vegetales que dotan al hombre del mayor número de plantas útiles para sus múltiples actividades cotidianas. En el mundo se encuentran 600 géneros de leguminosas con 11.000 especies de las cuales 25 son importantes. De las 11.000 especies sólo 600 son de origen tropical las demás son de la zona templada (Ministerio De Agricultura Y Desarrollo Rural 1992).

Debido a sus diferentes pisos térmicos y a su biodiversidad, Colombia presenta un gran potencial en cuanto a la disponibilidad y variedad de recursos naturales, los cuales incluyen una gran variedad de especies de pastos y forrajes. El uso de estos materiales como biomasa lignocelulósica para la producción de biocombustibles, tiene varias ventajas como son: no habría una competencia con la producción de alimentos ya que pueden ser empleadas tierras marginales o degradadas por la agricultura, se usarían tierras no destinadas para el cultivo de alimentos, presentan un menor costo que las materias primas convencionales, pueden realizarse mezclas de variedades y además son recursos renovables y abundantes para la bioconversión a azúcares (Sánchez-macías y Rodríguez 2009).

Por otra parte, la problemática actual concerniente a los combustibles fósiles debido a la disminución de las reservas de este recurso no renovable y a los efectos nocivos sobre el medio ambiente causados por el uso de los mismos, conllevan también a la búsqueda de fuentes alternativas de energía renovable.

Este artículo presenta una revisión de las principales especies forrajeras existentes en nuestro país, considerando aspectos tales como rendimiento de producción, uso, ubicación, composición lignocelulósica y rendimiento teórico a etanol; con el fin de establecer cuáles son las especies o variedades potenciales que podrían emplearse para la producción de biocombustibles en Colombia.

\section{PRINCIPALES ESPECIES FORRAJERAS PARA LA PRODUCCIÓN DE COMBUSTIBLES}

Las importantes variedades existentes de especies forrajeras en Colombia tienen como uso principal la alimentación animal (especialmente ganado vacuno). Por lo tanto, para este uso específico, se hace importante que la especie forrajera cumpla con las siguientes características: especies perennes y con capacidad rebrotadora, adaptación al piso térmico donde será utilizada, elevada y uniforme producción de forraje todo el año, alto valor nutritivo, facilidad de propagación, tolerancia a plagas y enfermedades de importancia económica, competitividad de crecimiento con otras especies y persistencia a plagas y enfermedades (Ministerio De Agricultura Y Desarrollo Rural 1992).

Las gramíneas forrajeras constituyen la principal fuente de alimentación de los herbívoros ya que crecen de manera espontánea en la mayoría de los potreros. Se adaptan muy fácilmente a las variedades del clima y aportan la mayor parte de la materia seca y los carbohidratos consumidos por el animal. Pertenecen a la familia de las monocotiledóneas y algunas de sus características 
son: raíces poco profundas en la mayoría de las especies, tallos cilíndricos que presentan nudos, hojas alternadas con nervaduras paralelas, la base de la hoja por lo general envuelve al tallo y terminan en punta y las flores por lo general son espiguillas (Estrada Álvarez 2004). Las leguminosas, son plantas pertenecientes al grupo de las dicotiledóneas, son frecuentemente utilizadas para aumentar la porción proteica de la ración de los animales. Las leguminosas, son el grupo más importante después de las gramíneas, debido fundamentalmente a su valor forrajero y a la capacidad de enriquecimiento del suelo por fijación de nitrógeno atmosférico. Algunas de sus características son: poseen en su mayoría raíces profundas y nódulos nitrificantes, es decir, pequeños nódulos que fijan nitrógeno por medio de una relación simbiótica, a través de bacterias del genero Rhizobium, las hojas son anchas y por lo general compuestas de 3 o más foliolos, y las semillas casi siempre crecen dentro de una vaina o legumbre (Estrada Álvarez 2004).

Cada pasto requiere unas determinadas condiciones para su desarrollo óptimo: algunas especies son perennes, se adaptan en suelos de fertilidad media a baja, se adaptan a suelos alcalinos 0 ácidos, resisten sequías o inundaciones y pueden requerir suelos bien drenados, entre otras condiciones edafológicas apropiadas para su cultivo. Debido a los pisos térmicos que presenta Colombia, estas variedades forrajeras pueden adaptarse en diferentes lugares y bajo diferentes condiciones, aunque esto pueda afectar su rendimiento. Algunas de las especies forrajeras más comunes empleadas en Colombia, entre gramíneas y leguminosas son mostradas en la tabla 1.

Actualmente, ha crecido el interés global por dar un mejor aprovechamiento de los recursos disponibles, y particularmente, debido a la problemática de los combustibles fósiles, se ha estudiado el rendimiento de estos recursos como materiales potenciales para la producción de biocombustibles (Woodward 2006; Segura S., Echeverri, y Mejía 2008; Schmer et al. 2008; Keshwani y Cheng 2009; Faga, Wilkins, y Banat 2010; Bouton 2007; Guo et al. 2008), ya que estos, transforman eficientemente la energía proveniente del sol a energía disponible a través de la fotosíntesis. Además, se han desarrollado diferentes estudios en cuanto a la adaptación y mejoramiento de especies con el fin de mejorar los rendimientos obtenidos (Guo et al. 2008)(Arias y Hernández 2002).

Pastos como bermuda, switchgrass, rye grass y pasto elefante, han sido objeto de diferentes estudios (Morais et al. 2009; Xu et al. 2012; Xu, Wang, y Cheng 2011; Sharma, Lyons, y McRoberts 2011; McLaughlin y Adams Kszos 2005) debido al potencial de estos para su transformación en biocombustibles. Dentro de las características más relevantes consideradas para su explotación en países como estados Unidos e Irlanda, está el rendimiento de materia seca de estas variedades, reportándose rangos de rendimientos para pasto bermuda, ryegrass, switchgrass y pasto elefante de $6-27,10-12,12-19$ y $45-67 \mathrm{Mg} \mathrm{ha}^{-1}$ año $^{-1}$, respectivamente, los cuales están entre los rangos reportados para muchas de las especies existentes en Colombia.

Para considerar la viabilidad de emplear estas especies como materia prima lignocelulósica para la producción de biocombustibles, es importante tener en cuenta aspectos como ubicación, periodicidad del cultivo o de la cosecha, rendimiento y uso. La ubicación hace referencia a la disponibilidad geográfica de los diferentes materiales lignocelulósicos, y tiene implicaciones de tipo económico en cuanto al transporte de ésta hasta el lugar de procesamiento, o por el contrario, las zonas de mayor producción del material lignocelulósico de interés podrían ser el lugar adecuado para una posible ubicación de la planta de producción de biocombustibles (etanol y butanol).

Así mismo la periodicidad y el uso o aplicación que tienen este tipo de recursos, son factores que afectan la viabilidad de la utilización de estos como materias primas. La periodicidad, se refiere al periodo de tiempo que demora obtener cierta cantidad de material lignocelulósico, más específicamente, cada cuanto tiempo se tiene materia prima disponible para el proceso. Además, es importante tener presente otras aplicaciones que puedan tener, de modo que no se vean afectados otros sectores por la implementación de este tipo de materiales en nuevos procesos, como por ejemplo la seguridad alimentaria, el ecosistema, el costo o la productividad en otros sectores industriales, además de la potencial competencia de mercados que pueda conducir a un incremento significativo de la materia prima que amenace la viabilidad económica del proceso. 
Tabla 1: Producción y uso de algunos pastos y forrajes en Colombia.

\begin{tabular}{|c|c|c|c|c|}
\hline Especie & Nombre científico & msnm & Rendimiento & Uso \\
\hline Gordura & Mellinis minutiflora & $200-2.200$ & $\begin{array}{l}5-20 \text { t/ha/año de materia } \\
\text { seca }\end{array}$ & $\begin{array}{c}\text { Corte, pastoreo, heno } \\
\text { y ensilaje }\end{array}$ \\
\hline Brachiaria & $\begin{array}{c}\text { Brachiaria } \\
\text { Decumbens }\end{array}$ & $0-2000$ & 20 t/ha/año de forraje seco & $\begin{array}{c}\text { Corte, pastoreo, heno } \\
\text { y ensilaje }\end{array}$ \\
\hline $\begin{array}{l}\text { Pasto } \\
\text { alambre }\end{array}$ & Brachiaria brizantha & $0-3000$ & $\begin{array}{l}10 \text { a } 17 \mathrm{Kg} / \mathrm{ha} / \mathrm{año} \text { de } \\
\text { materia seca }\end{array}$ & $\begin{array}{c}\text { Pastoreo, henificación } \\
\text { y ensilaje }\end{array}$ \\
\hline $\begin{array}{l}\text { Brachiaria } \\
\text { humidicola }\end{array}$ & $\begin{array}{l}\text { Brachiaria } \\
\text { humidicola }\end{array}$ & $\begin{array}{c}1000- \\
2000\end{array}$ & 10 t/ha/año de materia seca & $\begin{array}{c}\text { Pastoreo, ensilaje y } \\
\text { henificación }\end{array}$ \\
\hline $\begin{array}{l}\text { Pará, } \\
\text { Admirable }\end{array}$ & Brachiaria Mutica & $0-1.800$ & $\begin{array}{l}60 \text { t/ha/año en forraje verde } \\
\text { en } 6 \text { cortes }\end{array}$ & $\begin{array}{l}\text { Pastoreo rotacional, } \\
\text { heno }\end{array}$ \\
\hline Pasto Bufel & Cenchrus ciliaris & $0-2.000$ & $\begin{array}{l}60 \text { t/ha/ año, en estado } \\
\text { verde en } 6 \text { cortes }\end{array}$ & Pastoreo \\
\hline $\begin{array}{l}\text { Pasto } \\
\text { bermuda }\end{array}$ & Cynodon Dactylon & $0-1.800$ & $\begin{array}{l}1000 \text { a } 3000 \mathrm{~kg} / \mathrm{ha} / \mathrm{mes} \mathrm{de} \\
\text { materia seca }\end{array}$ & Pastoreo \\
\hline $\begin{array}{l}\text { Pasto } \\
\text { Estrella }\end{array}$ & $\begin{array}{c}\text { Cynodon } \\
\text { Plectostachium }\end{array}$ & $0-1.700$ & $\begin{array}{l}1 \text { a } 3 \text { t/ha/mes de materia } \\
\text { seca, en época seca }\end{array}$ & $\begin{array}{l}\text { Pastoreo, heno, } \\
\text { ensilaje y mezclas }\end{array}$ \\
\hline $\begin{array}{l}\text { Pasto } \\
\text { Angleton }\end{array}$ & $\begin{array}{l}\text { Dychanthium } \\
\text { Aristatum }\end{array}$ & $0-2.000$ & $\begin{array}{l}100-150 \text { t/ha/año en estado } \\
\text { verde }\end{array}$ & Pastoreo, corte \\
\hline $\begin{array}{l}\text { Pasto } \\
\text { Pangola }\end{array}$ & Digitaria Decumbens & $0-1.000$ & $\begin{array}{l}100 \text { t/ha/año en estado } \\
\text { verde }\end{array}$ & $\begin{array}{c}\text { Pastoreo rotacional, } \\
\text { heno }\end{array}$ \\
\hline $\begin{array}{l}\text { Pasto } \\
\text { Alemán }\end{array}$ & $\begin{array}{l}\text { Echinochloa } \\
\text { polystachia }\end{array}$ & $0-1.200$ & $\begin{array}{l}12-20 \text { t/ha/corte de forraje } \\
\text { verde }\end{array}$ & Corte y ensilaje \\
\hline $\begin{array}{l}\text { Pasto } \\
\text { Guinea }\end{array}$ & Pannicum maximun & $0-1.800$ & 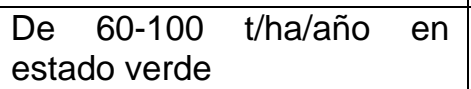 & $\begin{array}{l}\text { Corte, pastoreo y } \\
\text { ensilaje }\end{array}$ \\
\hline $\begin{array}{l}\text { Dallis, } \\
\text { Gramalote }\end{array}$ & Paspalum Dilatatum & $0-2.000$ & 20 t/ha/año en estado verde & $\begin{array}{c}\text { Corte, pastoreo , heno } \\
\text { y ensilaje }\end{array}$ \\
\hline $\begin{array}{l}\text { Bahía } \\
\text { común }\end{array}$ & Paspalum Notatun & $0-2.300$ & $\begin{array}{l}\text { 4-6 } \mathrm{t} / \mathrm{ha} / \text { corte } \text { en estado } \\
\text { verde }\end{array}$ & Pastoreo \\
\hline $\begin{array}{l}\text { Pasto } \\
\text { elefante }\end{array}$ & $\begin{array}{l}\text { Pennisetum } \\
\text { purpureum }\end{array}$ & $0-2.000$ & $\begin{array}{l}80-100 \text { t/ha/corte (cada } 10- \\
12 \text { semanas) }\end{array}$ & $\begin{array}{l}\text { Corte eventualmente } \\
\text { para ensilaje }\end{array}$ \\
\hline $\begin{array}{l}\text { Sorgo } \\
\text { forrajero }\end{array}$ & Sorghum vulgare & $0-1.800$ & $\begin{array}{l}30-40 \text { t/ha/corte en estado } \\
\text { verde (cortes 4-5/año) }\end{array}$ & Corte y ensilaje \\
\hline $\begin{array}{l}\text { Puntero, } \\
\text { Jaraguá }\end{array}$ & Hyparrehnia rufa & $0-1.500$ & 75 t/ha/año en estado verde & $\begin{array}{l}\text { Pastoreo, Heno y } \\
\text { ensilaje }\end{array}$ \\
\hline $\begin{array}{l}\text { Pasto } \\
\text { panamá }\end{array}$ & Saccharum sinense & $0-2.200$ & $48-50 \mathrm{t} / \mathrm{ha} /$ corte & Corte y ensilaje \\
\hline Alfalfa & Medicago sativa & $700-2.800$ & $\begin{array}{l}120 \text { t/ha/año en estado } \\
\text { verde }\end{array}$ & $\begin{array}{l}\text { Corte, pastoreo, heno, } \\
\text { ensilaje y harina }\end{array}$ \\
\hline Guandul & Cajanus cajan & $0-1.800$ & $\begin{array}{l}45 \text { a } 60 \text { t/año en estado } \\
\text { verde }\end{array}$ & Corte, ensilaje \\
\hline $\begin{array}{l}\text { Bejuco de } \\
\text { chivo, centro }\end{array}$ & $\begin{array}{l}\text { Centrosema spp- } \\
\text { pubescens. plumieri }\end{array}$ & $0-1.600$ & 12 t/ha de materia seca & $\begin{array}{c}\text { Corte, pastoreo, heno } \\
\text { y ensilaje }\end{array}$ \\
\hline $\begin{array}{l}\text { Acacia } \\
\text { forrajera }\end{array}$ & $\begin{array}{c}\text { Leucaena } \\
\text { leucocephala }\end{array}$ & $0-1.800$ & $\begin{array}{l}100 \text { t/ha/año en estado } \\
\text { verde }\end{array}$ & $\begin{array}{c}\text { Corte, ramoneo, } \\
\text { cercas vivas }\end{array}$ \\
\hline $\begin{array}{l}\text { Kudzú } \\
\text { tropical }\end{array}$ & $\begin{array}{c}\text { Pueraria } \\
\text { phaseoloides }\end{array}$ & $0-2.000$ & 54 t/ha/año en estado verde & $\begin{array}{l}\text { Corte, pastoreo, heno, } \\
\text { ensilaje, cultivo de } \\
\text { cobertura }\end{array}$ \\
\hline Kikuyo & $\begin{array}{l}\text { Pennisetum } \\
\text { clandestinum }\end{array}$ & $\begin{array}{l}2200- \\
3000\end{array}$ & $\begin{array}{l}80-100 \text { t/ha/año en estado } \\
\text { verde }\end{array}$ & pastoreo \\
\hline Climacuna & $\begin{array}{l}\text { Dychanthium } \\
\text { Annulatum }\end{array}$ & $0-1800$ & $\begin{array}{l}40-50 \text { t/ha/año de forraje } \\
\text { verde }\end{array}$ & pastoreo y ensilaje \\
\hline King grass & $\begin{array}{l}\text { pennisetum } \\
\text { hybridum }\end{array}$ & $0-2100$ & $\begin{array}{l}40-60 \text { t/ha/corte de forraje } \\
\text { verde cada } 45-60 \text { días }\end{array}$ & Corte y ensilaje \\
\hline
\end{tabular}

Considerando estos aspectos mencionados, se realizó una revisión sobre las especies colombianas más comunes y de las cuales se pudo encontrar la información requerida para poder realizar una comparación cualitativa (no mostrada) sobre las diferentes características que podrían 
potenciar la producción de distintas variedades de este tipo de biomasa para la producción de biocombustibles como etanol y butanol, claro está, en zonas degradadas por la agricultura y en tierras subutilizadas sin detrimento de la seguridad alimentaria humana o animal. En la tabla 1 se presentan la ubicación en metros sobre el nivel del mar (msnm), rendimiento de producción por área cultivada y por año, y usos de algunas de las especies forrajeras más comunes en Colombia, de acuerdo a una revisión sobre reportes y datos existentes concernientes a la producción de diferentes variedades de pastos y forrajes de uso en común en Colombia. Sin embargo, la información existente al respecto es escasa y sin actualización.

De acuerdo entonces a los datos disponibles para el rendimiento del cultivo, ubicación con respecto a la altura sobre el nivel del mar de pastos como elefante, king grass y sorgo forrajero, estas materias primas podrían convertirse en opciones potenciales para su transformación bioquímica en biocombustibles. Por otro lado, es importante anotar que los pisos térmicos sobre los cuales puede darse la ubicación de cultivos de estas especies constituye el $94.87 \%$ (Instituto Geografico Agustin Codazzi 2002) del territorio Colombiano y además, existen más de 35 millones de hectáreas disponibles para la agricultura y plantaciones forestales (Proexport Colombia 2010), lo cual justifica la implementación de pastos y otras especies forrajeras como cultivos energéticos en diferentes zonas del país, al igual que en otros países tropicales con características climáticas y edafológicas similares.

\section{COMPOSICIÓN LIGNOCELULÓSICA DE PASTOS Y FORRAJES}

La composición de los materiales lignocelulósicos es el factor que define la viabilidad del procesamiento fisicoquímico para la producción de etanol y butanol, pues de acuerdo a su contenido de lignina, celulosa y hemicelulosa se definen las condiciones bajo las cuales serán desarrolladas las etapas correspondientes al pretratamiento, hidrólisis y fermentación.

Las células vegetales se caracterizan por tener una pared que les da rigidez y estructura. Las plantas en estado vegetativo temprano tienen una sola capa en su pared celular y de poco espesor (pared primaria). A medida que la planta va madurando, se deposita una segunda capa interna llamada pared secundaria. Los principales componentes de estas dos paredes son la celulosa, hemicelulosa y lignina. Entre ambas pueden formar un 40 a $80 \%$ de la composición total del forraje El contenido de lignina en los pastos varía según la edad y especie, estando en un rango de $2 \%$ en la hierba joven hasta un $15 \%$ en la madura. El contenido de hemicelulosa varía ampliamente en las plantas, en un rango entre $6 \%$ a $40 \%$ de la materia seca, y la celulosa que es el componente más importante de la pared celular, se encuentra en un rango de 15 a $50 \%$ de la materia seca (Briceño 2002).

La composición y el rendimiento de los pastos y forrajes dependen del estado de desarrollo de la planta, del clima y del suelo, además de la variedad de la especie. En la tabla 2 se muestran los contenidos de lignina, celulosa y hemicelulosa de algunas especies de pastos y forrajes existentes en Colombia, calculadas con base a la materia seca.

\section{RENDIMIENTO TEÓRICO A ETANOL}

El contenido de celulosa y hemicelulosa, dan una idea de la cantidad de etanol que puede obtenerse a partir de diferentes materias primas. Este rendimiento teórico a etanol se calcula con base en las cantidades de xilosa y glucosa contenidas en los diferentes materiales (promedio de los datos mostrados en la tabla 3). En la tabla 2 se presentan los datos obtenidos para diferentes pastos y forrajes calculados estequiométricamente a partir de las reacciones químicas mostradas en las ecuaciones 1 y 2 , y de acuerdo al algoritmo mostrado en la ecuación 3.

\section{Xilosa}

$\mathrm{C}_{5} \mathrm{H}_{10} \mathrm{O}_{5} \stackrel{\text { microorganismo }}{\longrightarrow} 5 / 3 \mathrm{CO}_{2}+5 / 3 \mathrm{C}_{2} \mathrm{H}_{6} \mathrm{O}$ 
Tabla 2: Composición de Lignina, Celulosa y Hemicelulosa y rendimiento a etanol de algunos pastos y forrajes.

\begin{tabular}{|c|c|c|c|c|c|}
\hline Especie & $\%$ Lignina & $\%$ Celulosa & $\%$ Hemicelulosa & Etanol (L/Ton seca) & Referencia \\
\hline Brachiaria & $4,3-8,5$ & $29,2-34,3$ & $27,5-29,7$ & 440,52 & (Estrada Álvarez 2004) \\
\hline Pasto alambre & $4,9-10,1$ & $24,4-34,6$ & $19,9-42,3$ & 442,89 & (Estrada Álvarez 2004) \\
\hline $\begin{array}{l}\text { Brachiaria } \\
\text { humidicola }\end{array}$ & 10,4 & 37,7 & 37,0 & 545,49 & $\begin{array}{c}\text { (Arias y Hernández } \\
\text { 2002) }\end{array}$ \\
\hline $\begin{array}{l}\text { Pará, } \\
\text { Admirable }\end{array}$ & $3,4-7,1$ & $22,9-37,3$ & $25,8-36,1$ & 446,63 & (Estrada Álvarez 2004) \\
\hline Pasto Bufel & 4,5 & 26,9 & 33,1 & 438,90 & (Palma 2003) \\
\hline $\begin{array}{l}\text { Pasto } \\
\text { bermuda }\end{array}$ & 11,9 & 20,4 & 31,0 & 376,30 & (Delgadillo Puga 2001) \\
\hline Pasto Estrella & $4,0-5,4^{*}$ & $22,5-31,8$ & $32,0-35,7$ & 446,25 & (Briceño 2002) \\
\hline $\begin{array}{l}\text { Pasto } \\
\text { Angleton }\end{array}$ & $5,3-11,8$ & $32,4-39,0$ & $21,9-23,6$ & 426,42 & (Estrada Álvarez 2004) \\
\hline Pasto Pangola & $6,4-8,3$ & $32,1-33,6$ & $29,0-32,0$ & 465,60 & $\begin{array}{c}\text { (Rodríguez-Carrasquel, } \\
\text { Chicco, y Chacon } \\
1997)\end{array}$ \\
\hline $\begin{array}{l}\text { Pasto Guinea, } \\
\text { India }\end{array}$ & $4,3-6,2$ & $31,3-33,8$ & $20,4-26,3$ & 407,63 & (Estrada Álvarez 2004) \\
\hline Pasto elefante & $7^{*}$ & 36 & 28 & 466,91 & (Soest 1994) \\
\hline $\begin{array}{l}\text { Sorgo } \\
\text { forrajero }\end{array}$ & 2,97 & 26,7 & 20,9 & 347,35 & $\begin{array}{l}\text { (Amador y Boschini } \\
2000)\end{array}$ \\
\hline Pasto panamá & 13,6 & 34,4 & 27,2 & 193,14 & $\begin{array}{c}\text { (García De H. et al. } \\
1994)\end{array}$ \\
\hline Alfalfa & 8,7 & 21,2 & 5,4 & 215,18 & (Faner 2007) \\
\hline Guandul & 28,5 & 29,8 & & 305,28 & (Arias Arguello 1990) \\
\hline $\begin{array}{l}\text { Acacia } \\
\text { forrajera }\end{array}$ & $7,15-11,5$ & $16,5-18,2$ & $19,9-29,0$ & 255,58 & $\begin{array}{c}\text { (García De H. et al. } \\
1994)\end{array}$ \\
\hline Kudzú tropical & 18,2 & 27,4 & $6,28-9,3$ & 387,90 & (Estrada Álvarez 2004) \\
\hline kikuyo & 5,6 & 26,9 & 26,2 & 68,60 & $\begin{array}{c}\text { (Correa C, Pabón R, y } \\
\text { Carulla F 2008) }\end{array}$ \\
\hline Matarratón & 15,4 & 9,5 & & 366,01 & (Arias Arguello 1990) \\
\hline climacuna & 4,1 & 26,1 & 24,0 & 442,89 & (Estrada Álvarez 2004) \\
\hline king grass & 6,4 & 32,6 & 25,9 & 440,52 & (Estrada Álvarez 2004) \\
\hline
\end{tabular}

\section{Glucosa}

$\mathrm{C}_{6} \mathrm{H}_{12} \mathrm{O}_{6} \stackrel{\text { microorganismo }}{\longrightarrow} 2 \mathrm{CO}_{2}+2 \mathrm{C}_{2} \mathrm{H}_{6} \mathrm{O}$

$E \operatorname{tanol}(L /$ Ton sec $a)=\frac{\frac{X_{H C}{ }^{*} 1.11 * v_{x e}{ }^{*} M_{\text {etOH }}}{M_{x i l}}+\frac{X_{C} * 1.11 * v_{G e}{ }^{*} M_{e t O H}}{M_{G}} * 10000}{\rho_{\text {etoH }}}$

Donde:

$X_{H C}$ : es la fracción de hemicelulosa en 100 gramos de material seco

$X_{C}$ : es la fracción de celulosa en 100 gramos de material seco

1.11: es la relación entre el peso molecular de la celulosa y la celobiosa

$\mathrm{v}_{\mathrm{x} e}$ : factor estequiométrico de la conversión de xilosa en etanol

$\mathrm{v}_{\mathrm{Ge}}$ : factor estequiométrico de la conversión de glucosa en etanol 
$M_{\text {etOH: }}$ peso molecular del etanol

$M_{\text {xil: }}$ : peso molecular de la xilosa

$M_{G}$ : peso molecular de la glucosa

10000: factor de conversión de $100 \mathrm{~g}$ a toneladas

$\rho_{\text {etoH: }}$ densidad del etanol $(\mathrm{g} / \mathrm{L})$

En el caso del rendimiento teórico a etanol, las especies Brachiaria humidicola, Pennisetum purpureum y Digitaria Decumbens presentan los mayores rendimientos, por lo cual son especies con potencial para la obtención tanto de bioetanol como de biobutanol.

A nivel de laboratorio, los resultados obtenidos en nuestros estudios sobre la fermentabilidad de los pastos elefante y king grass pretratados mediante hidrólisis alcalina con $\mathrm{NaOH}$ a $120^{\circ} \mathrm{C}$, usando levadura comercial Saccharomyces cerevisiae Ethanol Red, mostraron una producción de etanol referida a la materia prima alimentada al proceso de $191.7 \mathrm{~L} /$ Ton material seco y $195.4 \mathrm{~L}$ /Ton material seco, respectivamente, lo cual corresponde a $78.7 \%$ del rendimiento teórico para el king grass y al $73.7 \%$ del rendimiento teórico para el pasto elefante, para la fermentación de la fracción celulósica del material.

Tabla 3: Especies forrajeras potenciales para la producción de bioetanol y biobutanol.

\begin{tabular}{|c|c|c|c|c|c|c|}
\hline \multirow[b]{2}{*}{ Especie } & \multirow[b]{2}{*}{ Nombre científico } & \multicolumn{3}{|c|}{ Composición } & \multirow[b]{2}{*}{ Rendimiento } & \multirow{2}{*}{$\begin{array}{l}\text { Rendimiento } \\
\text { a etanol } \\
\text { (L/Ton seca) }\end{array}$} \\
\hline & & \% Lignina & \% Celulosa & $\%$ Hemicelulosa & & \\
\hline $\begin{array}{l}\text { Pasto } \\
\text { elefante }\end{array}$ & $\begin{array}{l}\text { Pennisetum } \\
\text { purpureum }\end{array}$ & 7 & 36 & 28 & $\begin{array}{c}80-100 \text { t/ha/corte } \\
\text { (cada 10-12 } \\
\text { semanas) }\end{array}$ & 466,91 \\
\hline King grass & $\begin{array}{l}\text { pennisetum } \\
\text { hybridum }\end{array}$ & 13,59 & 34,38 & 27,25 & $\begin{array}{l}40-60 \mathrm{t} / \mathrm{ha} / \text { corte } \\
\text { de forraje verde } \\
\text { cada } 45-60 \text { días }\end{array}$ & 449,67 \\
\hline pasto aguja & $\begin{array}{l}\text { Brachiaria } \\
\text { humidicola }\end{array}$ & 10,39 & 37,7 & 36,97 & $\begin{array}{l}10 \text { t/ha/año de } \\
\text { materia seca }\end{array}$ & 545,49 \\
\hline $\begin{array}{c}\text { Pasto } \\
\text { panamá, } \\
\text { caña } \\
\text { japonesa }\end{array}$ & $\begin{array}{l}\text { Saccharum } \\
\text { sinense }\end{array}$ & 13,59 & 34,38 & 27,25 & $48-50 \mathrm{t} / \mathrm{ha} /$ corte & 449,67 \\
\hline
\end{tabular}

\section{CONCLUSIONES}

Colombia posee una gran variedad de especies que podrían emplearse como materias primas en la producción de biocombustibles. Gracias a la variedad de pisos térmicos pueden cultivarse estas variedades en muchas zonas del país, lo cual sería una gran ventaja en cuanto a la disponibilidad de los recursos, al igual que para el transporte y almacenamiento de materias primas.

Las especies forrajeras que se consideran materiales lignocelulósicos potenciales para la producción de etanol, son aquellos que tienen las mejores características de rendimiento de producción de forraje, ubicación en el territorio colombiano, composición lignocelulósica, uso y periodicidad. Por lo tanto, dentro de las especies mencionadas en esta revisión, las que se consideran más adecuadas para su exploración en la producción de bioetanol se presentan en la tabla 3.

\section{REFERENCIAS}

Amador, A. y C. Boschini, Calidad nutricional de la planta de sorgo negro forrajero (sorghum almum) para alimentación animal, Agronomía mesoamericana: 11, 79-84 (2000). 
Arias A. y H. Hernández, Composición química del pasto aguja (brachiaria humidícola) sometida a pastoreo en una finca del municipio Guanare estado portuguesa, Revista Científica: 12, 562-565 (2002).

Arias $\mathrm{H} .$, Cambios en la composición bioquímica y su aplicabilidad en el uso de follajes verdes como fuente de materia orgánica y nutrimentos en sistemas agroforestales, Agronomía Colombiana: 12, 169-181 (1995).

Barba P., Síntesis de carboximetilcelulosa (CMC) a partir de pastas de plantas anuales, http://tdx.cat/handle/10803/8503. Acceso: 10 de junio (2011).

Bernal Eusse, J., Pastos y Forrajes Tropicales, 3 edición, 50-575. Banco Ganadero, (1994).

Boschini, C., H. Dormond, y A. Castro, Composición química de la morera (morus alba), para uso en la alimentación animal: densidades y frecuencias de poda. Agronomía mesoamericana: 11, 4149 (2000).

Bouton J.H., Molecular breeding of switchgrass for use as a biofuel crop. Current Opinion in Genetics \& Development: 17,553-558 (2007).

Briceño G.E., Aspectos cualitativos y cuantitativos de los factores que afectan la digestabilidad ruminal de los forrajes tropicales. Facultad de medicina Veterinaria y Zootecnia. Universidad Autónoma de Yucatán, (2002).

Cerrillo, M.A., O. López, y A. Juárez-Reyes, Composición química y producción de gas in vitro de cactáceas, arbustivas y arbóreas presentes en la dieta de caprinos en pastoreo, (2004), http://ammveb.net/XXVI\%20CNB/memorias/rum/docs/rum11.doc. Acceso: 1de junio 2011.

Combellas J., E. Gonzáles, y R. Parra, Composición y valor nutritivo de forrajes producidos en el trópico. I: digestibilidad aparente y verdadera de las fracciones químicas, Agronomía Tropical: 21, 483-494 (1971).

Correa H.J., M.L. Pabón, y J.E. Carulla., Valor nutricional del pasto kikuyo (Pennisetum clandestinum Hoechst Ex Chiov.) para la producción de leche en Colombia (Una revisión): I Composición química y digestibilidad ruminal y posruminal, Livestock Research for Rural Development: 20, (2008).

Cuesta P. A., Producción y utilización de recursos forrajeros en sistemas de producción bovina de las regiones Caribe y valles interandinos, Publicación de Corpoica, 108, (2005).

Chen F. y R.A. Dixon, Lignin modification improves fermentable sugar yields for biofuel production. Nature Biotechnology: 25, 759-761 (2007).

Delgadillo C., Efecto de la complementación alimenticia de gramíneas tropicales con un alimento complejo catalítico sobre las variables de fermentación ruminal en bovinos y ovinos, 130, (2001).

Estrada A., J., Pastos y forrajes para el trópico colombiano, Manizales: Universidad de Caldas, (2004).

Faga B.A., M.R. Wilkins y I.M. Banat, Ethanol production through simultaneous saccharification and fermentation of switchgrass using Saccharomyces cerevisiae D5A and thermotolerant Kluyveromyces marxianus IMB strains, Bioresource Technology: 101, 2273-2279 (2010).

Faner C.L. La pastura de alfalfa como fuente de alimentación para cerdos en crecimiento y terminación, (2007), http://www.produccion-animal.com.ar/produccion_porcina/72-alfalfa.pdf. Acceso: 1 de junio de 2010. 
Fu C., J.R. Mielenz, X. Xiao, Y. Ge, C.Y. Hamilton y M. Rodriguez, Genetic manipulation of lignin reduces recalcitrance and improves ethanol production from switchgrass, PNAS: 108, 3803-3808 (2011).

García M., C. Sanchéz, J. Colmenarez, y E. Beltrán, Suplementación a pastoreo de leucaena leucocephala en vacas mestizas de doble propósito en el valle de Aroa, Venezuela, Zootecnia Trop: 12, 205-224 (1994).

Guerrero R. Fertilización de pastos tecnificados, (1995), http://hasp.axesnet.com/contenido/documentos/Medcapitulo14.pdf. Acceso: 8 junio de 2010

Guo G., W. Chen, L. Men y W. Hwang, Characterization of dilute acid pretreatment of silvergrass for ethanol production. Bioresource Technology: 99, 6046-6053 (2008).

Hisano H., R. Nandakumar, y Z. Wang, Genetic modification of lignin biosynthesis for improved biofuel production, Plant Cell, 306-313 (2009).

Instituto Geografico Agustin Codazzi, Corporación Colombiana De Investigación Agropecuaria. Corpoica. Zonificación de los conflictos de uso de las tierras en Colombia. 2002. Zonificación de los conflictos de uso de las tierras en Colombia. Bogotá D. C.

Keshwani D.R. y J.J. Cheng, Switchgrass for bioethanol and other value-added applications: $A$ review, Bioresource Technology: 100, 1515-1523 (2009).

McLaughlin, Samuel B. y L. A. Kszos, Development of switchgrass (Panicum virgatum) as a bioenergy feedstock in the United States, Biomass and Bioenergy: 28,6, 515-535 (2005) .

Ministerio De Agricultura Y Desarrollo Rural, La Ganadería en Colombia Pastos y forrajes bovinos: (1992). http://www.cyemh.org/ganaderiaencolombia.htm.

De Morais, R. F., B. J. De Souza, J. M. Leite, L. H., y De B. Soares, Elephant grass genotypes for bioenergy production by direct biomass combustion, Pesq. Agropec. bras., Brasília: 4, 133-140 (2009).

Palma J.M., Los sistemas silvopastoriles en el trópico seco Mexicano, Archivos Latinoamericanos de Producción Animal: 14, 95-104, (2003).

Proexport Colombia. Sector Agroindustrial Colombiano, Invierta en Colombia trabajo compromiso ingenio: 1, 1, 1-17 (2010).

Provet. Pastos y forrajes.

http://www.laboratoriosprovet.com.co/LinkClick.aspx?fileticket=1JuNfP9KvDs\%3D\&tabid=78\&lang uage=es-CO. Acceso: 8 de junio de 2010.

Ralph J., B.A. Simmons y D. Loque, Advances in modifying lignin for enhanced biofuel production, Current Opinion in Plant Biology: 13, 313-320 (2010).

Rodríguez-Carrasquel S., C.F. Chicco, y E. Chacon, Efecto de la aspersión de urea sobre el rendimiento, composición química y digestibilidad del pasto pangola Y A-24, Agronomía Tropical: 24, 183-192 (1997).

Sánchez-Macías, D.J.I., y F. Rodríguez, Expectativas Del Sector De La Bioenergía en Castilla y León. (2009).

Schmer M., K. Vogel, R. Mitchell y R. Perrin, Net energy of cellulosic ethanol from switchgrass, PNAS: 105, 464-469 (2008). 
Segura F., R. Echeverri R., y A. Mejía. Delignificación selectiva del pasto pennisetum purpureum x pennisetum typhoides usando basidiomicetos ligninolíticos, Vitae:15, 41-50, (2008).

Sharma, H.S. Shekhar, Gary Lyons, y Colin McRoberts, Biorefining of perennial grasses: $A$ potential sustainable option for Northern Ireland grassland production, Chem. Eng. Res. and Design: 89, 11, 2309-2321 (2011).

Soest, V. Nutritional ecology of the ruminant, 2 edición, Cornell University Press, (1994).

Sun Y. y J.J. Cheng., Dilute acid pretreatment of rye straw and bermudagrass for ethanol production, Bioresource Technology: 96, 1599-160, (2005).

Urquiaga S., G. Cadisch, B.J. Alves, R.M. Boddey y K.E. Giller, Influence of decomposition of roots of tropical forage species on the availability of soil nitrogen, Soil Biol. Biochem: 30, 20992106, (1998).

Woodward W.T. The Potential of Alfalfa, Switchgrass and Miscanthus As Biofuel Crops in Washington, Energy: 1-7, (2006).

Xu, J., X. Zhang, R. R Sharma-Shivappa, y M. W. Eubanks, Gamagrass varieties as potential feedstock for fermentable sugar production, Bioresource technology: 116, 540-544 (2012).

$\mathrm{Xu}$, J., Z. Wang, y J. J Cheng, Bermuda grass as feedstock for biofuel production: a review, Bioresource technology: $102,17,7613-7620$ (2011).

Yang S., I. Kataeva, S.D. Hamilton-Brehm, N.L. Engle, T.J. Tschaplinski, C. Davis, J. Westpheling, y M.W. Adams, Efficient Degradation of Lignocellulosic Plant Biomass, without Pretreatment, by the Thermophilic Anaerobe. Anaerocellum thermophilum DSM 6725. Applied and Environmental Microbiology: 75, $4762-4769$ (2009). 